\title{
Anthropogenic pressure on the largest lakes of the River Tywa catchment
}

\author{
Jacek Kubiak $^{1^{*}}$, Sylwia Machula ${ }^{1}$, Dorota Oszkinis ${ }^{2}$, Dominik Rokicki ${ }^{1}$ \\ ${ }^{1}$ Department of Hydrochemistry and Aquatic Biological Resources, West Pomeranian University of Technology, Kazimierza Królewicza 4, \\ 71-550 Szczecin, Poland, e-mail: jacek.kubiak@zut.edu.pl (*corresponding author); sylwia.machula@zut.edu.pl \\ ${ }^{2}$ Eurobiznes, Boguchwały 8, 71-530 Szczecin, Poland
}

\begin{abstract}
The largest lakes of the River Tywa basin i.e. Strzeszowskie, Dołgie, Swobnica and Dłużec (north-west Poland, West Pomeranian Lakeland) were studied in the period 2008-2013, usually in a six week cycle. The aim of the study was to determine the degree of anthropogenic pressure on the lakes. Anthropogenic pressure was assessed against the natural vulnerability of lakes to degradation and the actual rate of eutrophication, with particular consideration of the catchment areas. Catchments of the studied lakes were classified as III class - catchment with moderate possibility of matter supply to lakes. Lakes Swobnica and Dłużec are characterised by low resistance to degradation. Lake Strzeszowskie is categorised as having moderate resistance and Lake Dołgie is non-resistant to degradation and highly susceptible to external pressure. The rate of eutrophication of lakes Strzeszowskie and Dłużec was found to be moderate, whereas lakes Dołgie and Swobnica show a high rate of eutrophication. The analysis of nutrient loading reaching the waters of the aforementioned lakes shows that the main area source is arable land, and the fundamental point source is the inflow of river waters to each of the analysed reservoirs. Nutrient loading supplied by the river is several times higher than area load. Such conditions require protective action to be taken within the catchment area and particularly, intensive implementation of good agricultural practice. In the studied lakes, there is a significant predominance of incoming nutrient loadings over dangerous and admissible loads.
\end{abstract}

Key words: lake eutrophication, nutrient loading, susceptibility to degradation, catchment influence

\section{Introduction}

Anthropogenic pressure intensifies the processes of aquatic environment transformation and degradation. This particularly applies to lakes which are excessively supplied with nutrients - they undergo eutrophication (Vollenweider 1968, 1976; Jonge et al. 2002; Kubiak and Tórz 2005). Urbanisation, industrialisation, intensive agricultural production and considerable tourism development coupled with insufficient methods of protection result in excessive anthropopressure and external loading with phosphorus and nitrogen (Vollenweider 1968; 1976; Lossow 1996, 1998, Kubiak 2003, Bechmann et al. 2005; Kubiak and Tórz 2006; Neal and Heathwaite 2005; Kato et al.2009). The load of these elements delivered to lakes often exceeds levels considered dangerous (Vollenweider 1968; Kubiak 2003; Neal and Heathwaite 2005; Kato et al. 2009). Intensive eutrophication leads to a significant decrease in the usefulness of water for the economy (industry, municipal services), recreation and sustainable fishery (Kubiak 2003; Kubiak and Tórz
2005; Kubiak et al. 2012; Górniak et al. 2016; Sieńska et al. 2016). Eutrophication as the most significant cause of disruption of lake ecosystems (Vollenweider 1989; Jonge et al. 2002) explicitly concerns the lakes of Western Pomerania (Kubiak 2003; Jarosiewicz et al. 2011, WIOŚ 2016).

Natural physio-geographical features of the direct catchment in particular can promote or limit matter load (including biogenic) delivered to water reservoirs (Olszewski and Tadajewski 1959; Bajkiewicz-Grabowska 2002; Neal and Heathwaite 2005). Lakes resistant to degradation have higher utility values (Olszewski 1971; Lossow 1996; Kubiak 2003). Identification of the causes and the rate of the change in trophic status of lakes would make it possible to determine the implementation of adequate preventive measures (Kubiak 2006; Sieńska et al. 2016).

Despite the natural and economic values of the lakes of the Tywa River, their multiannual cycles have not been analysed in so far. The lakes are characterised by a high degree of naturalness of plant community and 
low share of synanthropic species. The water reservoirs under analysis belong to the network of nature protection areas Natura 2000 (Site PLH320050 Dolina Tywy) (Raczyński and Waloch 2012).

The study concerns the lakes: Strzeszowskie, Dołgie, Swobnica and Dłużec. The following major environmental threats were identified: eutrophication, strong interference with the River Tywa ecosystem (fragmentation, disturbing water flow, periodic drying out of rivers, water and soil pollution) and forestry which does not always take into account the potential of the habitat and introduces ecologically and geographically alien species to the catchment area (e.g. spruce, larch, red oak) (Raczyński and Waloch 2012).

The aim of the present study was to determine the degree of anthropogenic pressure on the aforementioned lakes. Anthropogenic pressure was assessed against the natural vulnerability of lakes to degradation and the rate of eutrophication, with particular consideration of the catchment area.

The analyses conducted in the course of the research allow identification of the status of the lakes and methods of reversing or at least limiting or eliminating the negative effects of anthropogenic pressure. Furthermore, on the basis of the results of the research, potential uses and protection methods of particular reservoirs may be identified.

\section{Materials and methods}

The lakes of the River Tywa basin, i.e. Strzeszowskie, Dołgie, Swobnica and Dłużec (Fig. 1) were analysed in the period 2008-2013, usually in a six week cycle. Detailed morphometric data and hydrographical characterisation is presented in Table 1.

The vulnerability of the analysed lakes to degradation was evaluated using two systems: one by Bajkiewicz-Grabowska (2002), and the second according to Kudelska et al. (1994). The intensity of the effect of the catchment area on the lake was determined according to the model of Bajkiewicz-Grabowska (2002). Natural rate of eutrophication was identified by means of a comparison of the lake vulnerability to degradation with the role of the catchment area in supplying the lakes with matter (Bajkiewicz-Grabowska 2002). These methods have previously been used in a study on lakes of Western Pomerania (Kubiak 2003; Bajkiewicz-Grabowska et al. 2011).

Anthropogenic pressure of the catchment on the analysed lakes was assessed on the basis of the real nutrient loading supplied to the lakes (Bajkiewicz et al. 2011). Unit coefficients of the load delivered from the catchment were calculated using the data presented in the literature on the subject (MGGP and IOŚ 2010; Bajkiewicz

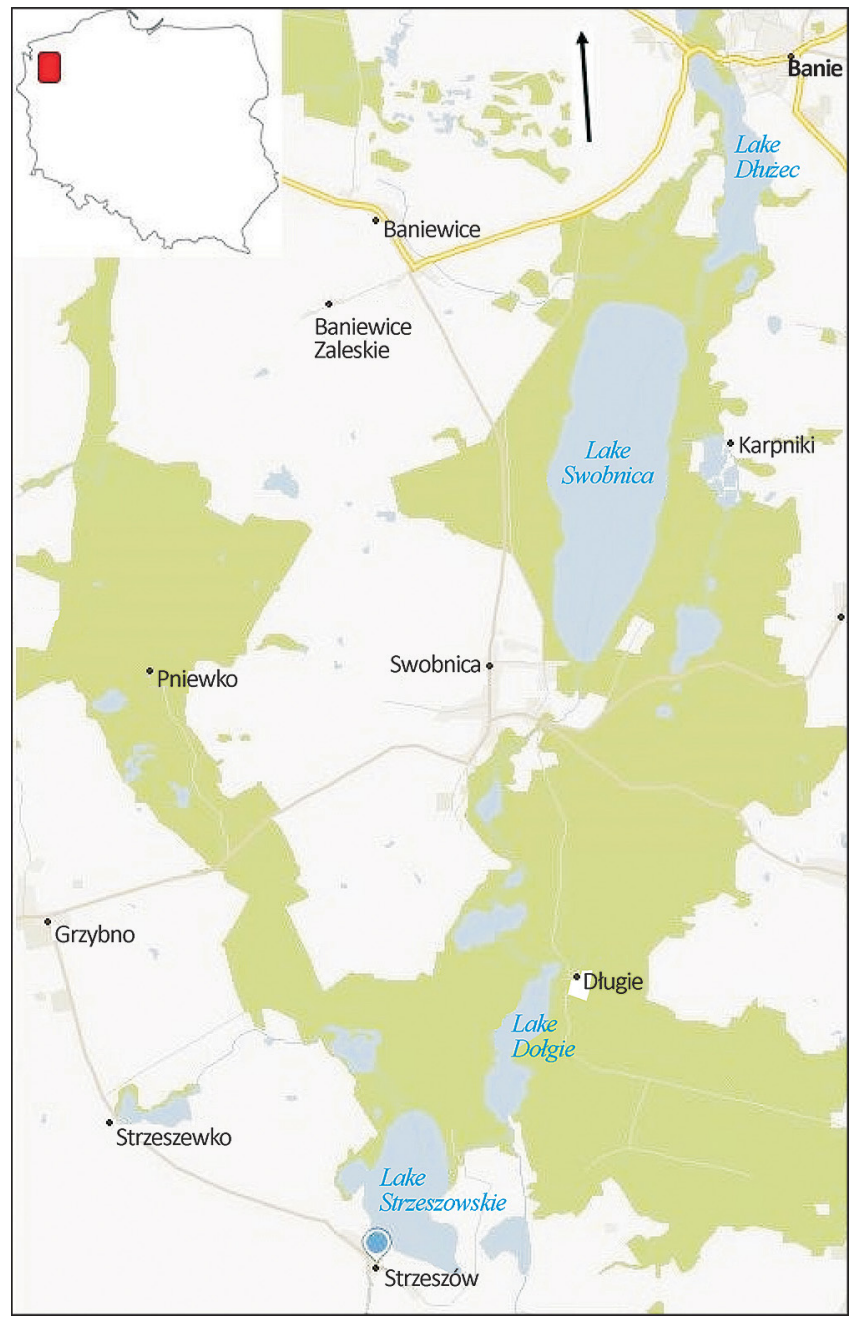

Fig. 1. Location of the studied lakes (www.mapy.google.pl)

et al. 2011). Threat of progression of lake eutrophication was assessed on the basis of the rate of eutrophication; the calculated real load was compared with the permissible and excessive load according to the hydrological model of Vollenweider (1968) which is widely used in limnology and has been adopted to investigate various groups of lakes in Poland (e.g. Uchmański and Szeligiewicz 1988; Kubiak 2003; MGGP and IOŚ 2010; Bajkiewicz-Grabowska et al. 2011; Jarosiewicz et al. 2011).

Land use in the total and direct catchment areas was identified using GIS tools (Geographical Information System) and land use maps (EEA 2006).

The main sources of biogenic substances were identified and methods of for the protection of particular reservoirs were shown (MGGP and IOŚ 2010; Bajkiewicz-Grabowska et al. 2011).

In the assessment of water quality of the individual lakes under analysis, widely applicable hydro-chemical indices were used (e.g. Kudelska et al. 1994; Kubiak 2003). 
Table 1. Morphometric parameters and hydrographical characteristic of the studied lakes

\begin{tabular}{|c|c|c|c|c|}
\hline \multirow{2}{*}{ Parameter/characteristic } & \multicolumn{4}{|c|}{ Lake } \\
\hline & Strzeszowskie & Dołgie & Swobnica & Dłużec \\
\hline Altitude & $52^{\circ} 59^{\prime} 35^{\prime \prime} \mathrm{N}$ & $53^{\circ} 00^{\prime} 25^{\prime \prime} \mathrm{N}$ & $53^{\circ} 03^{\prime} 50^{\prime \prime} \mathrm{N}$ & $53^{\circ} 05^{\prime} 34^{\prime \prime} \mathrm{N}$ \\
\hline Longitude & $14^{\circ} 35^{\prime} 31^{\prime \prime} \mathrm{E}$ & $14^{\circ} 37^{\prime} 41^{\prime \prime} \mathrm{E}$ & $14^{\circ} 38^{\prime} 18^{\prime \prime} \mathrm{E}$ & $14^{\circ} 39^{\prime} 36^{\prime \prime} \mathrm{E}$ \\
\hline Water region & \multicolumn{4}{|c|}{ Lower Oder River and Western Pomerania } \\
\hline Catchment & \multicolumn{4}{|c|}{ Rurzyca and Tywa rivers (SZ06) } \\
\hline River basin area (name and code) & \multicolumn{4}{|c|}{ Oder River basin / 6000} \\
\hline Combined surface water bodies & \multicolumn{4}{|c|}{ Tywa River (DO0605) } \\
\hline European surface water body code & PLLW11008 & PLLW11010 & PLLW11012 & PLLW11014 \\
\hline Balance type & flow-through & flow-through & flow-through & flow-through \\
\hline Assessment of the risk of failure to meet environmental objectives & unthreatened & unthreatened & unthreatened & unthreatened \\
\hline Maximum length $[\mathrm{m}]$ & 1900 & 1850 & 3900 & 2400 \\
\hline Maximum width [m] & 1150 & 450 & 1200 & 500 \\
\hline Length of the shoreline [m] & 5750 & 4350 & 9000 & 5875 \\
\hline Shoreline development index & 1.44 & 1.64 & 1.37 & 1.79 \\
\hline Water table area [ha] & 127.2 & 56.3 & 344 & 85.2 \\
\hline Volume $\left[\mathrm{dam}^{3}\right]$ & 9499 & 1700 & 14485 & 5228 \\
\hline Maximum depth [m] & 14.2 & 6.6 & 6.8 & 10.4 \\
\hline Depth ratio (mean/maximum) & 0.52 & 0.47 & 0.62 & 0.58 \\
\hline
\end{tabular}

The results were processed according to methodological guidelines using hydrological and morphometric data of the lakes under analysis as provided by the IMGW (Institute of Meteorology and Water Management) (Czarnecka et al. 1989), given by other authors (Filipiak and Sadowski 1994; Filipiak and Raczyński 2000) or calculated in the course of the present analysis.

\section{Results and discussion}

Each lake has a number of various individual features (e.g. morphometric, physio-geographical, particularly concerning the direct catchment) which determine the direction and intensity of transformation. The most vulnerable to degradation are the shallow lakes characterised by high rates of water exchange and considerable water mass dynamics (Kubiak 2003; Kubiak and Tórz 2006). Contemporary transformations of lake systems are determined by numerous relationships between natural tolerance and the intensity of environmental pressure. Excessive, usually anthropogenic, pressure of the catchment inevitably leads to permanent disruption of lake biogeocenosis which, in turn, results in rapid qualitative transformation of the aquatic environment, i.e. degradation (BajkiewiczGrabowska 2002; Bechmann et al. 2005; Borowiak and Maślanka 2007; Ptak et al 2013).

When defining environmental pressure on the lakes, it was found that the Lake Dołgie catchment is characterised by low capacity to mobilise the deposited load and that the possibility of the load reaching the reservoir is slight. However, the catchment areas of the remaining lakes are characterised by an average capacity to mobilise the deposited load and moderate capacity to supply matter.

The lakes under analysis were assessed in terms of susceptibility to degradation according to the classification by Bajkiewicz-Grabowska (2002). Lake Strzeszowskie is moderately resistant to degradation, the remaining lakes are characterised by low resistance to degradation. Similar results were obtained when the lakes were assessed according to the classification by Kudelska et al. (1994) - with the exception of Lake Dołgie which was identified as non-resistant (Tables 2-5).

Given the above, it was found that lakes Strzeszowskie and Dłużec are characterised by average susceptibility to degradation - class II of vulnerability, and the catchment areas of these reservoirs have high capacity to mobilise load - group 3 (Table 5). Furthermore, it was found that the aforementioned reservoirs undergo eutrophication at a moderate rate - type 2. Swobnica and Dołgie lakes are marked by a high rate of eutrophication due to high susceptibility to degradation (class III and IV), and the features of their catchment areas promote surface run-off. Such conditions greatly accelerate eutrophication (Bajkiewicz-Grabowska 2002) (Tables 2-5).

The same method was used by Bajkiewicz-Grabows$\mathrm{ka}$ (2002) in the assessment of the eutrophication rate of 177 lakes of various lakelands (Mrągowo Lakeland, Olsztyn Lakeland, Ełk Lakeland, Great Masurian Lakes) and the lakes of the Masurian Plain and Augustów Plain; and by Kubiak (2003) in the analysis of the greatest lakes of Western Pomerania. The aforementioned methods have also been used in studies on the assessment of the status of lakes in terms of meeting the re- 
Table 2. Characteristics of catchment areas as a source of biogenic matter supply according to Bajkiewicz-Grabowska (2002)

\begin{tabular}{|c|c|c|c|c|c|c|c|}
\hline \multirow{2}{*}{ Parameter } & \multirow{2}{*}{ Unit } & \multirow{2}{*}{ Catchment } & \multirow{2}{*}{ Value/score } & \multicolumn{4}{|c|}{ Lake } \\
\hline & & & & Strzeszowskie & Dołgie & Swobnica & Dłużec \\
\hline \multirow{2}{*}{ Lake density } & \multirow{2}{*}{ [\%] } & \multirow{4}{*}{ Total } & Value & 11.3 & 10.1 & 33.3 & 6.8 \\
\hline & & & Score & 1 & 1 & 1 & 0 \\
\hline \multirow{2}{*}{ Balance type ${ }^{1,2}$} & & & Value & flow-through & flow-through & flow-through & flow-through \\
\hline & & & Score & 3 & 3 & 3 & 3 \\
\hline \multirow{2}{*}{ River network density ${ }^{1}$} & \multirow{2}{*}[\mathrm{km}\mathrm{km}^{-2}]{} & \multirow{10}{*}{ Direct } & Value & 0.4 & 0.6 & 0.8 & 1.1 \\
\hline & & & Score & 0 & 1 & 1 & 2 \\
\hline \multirow{2}{*}{ Mean catchment slope } & \multirow{2}{*}{ [\%] } & & Value & 3.3 & 3.9 & 4.3 & 4.3 \\
\hline & & & Score & 0 & 0 & 0 & 0 \\
\hline \multirow{2}{*}{ Closed drainage areas ${ }^{2}$} & \multirow{4}{*}{ [\%] } & & Value & 0.0 & 0.0 & 0.0 & 0.0 \\
\hline & & & Score & 3 & 3 & 3 & 3 \\
\hline \multirow{2}{*}{ Catchment geology ${ }^{2}$} & & & Value & sandy-clay & sandy-clay & sandy-clay & sandy-clay \\
\hline & & & Score & 2 & 2 & 2 & 2 \\
\hline \multirow[t]{2}{*}{ Catchment use } & & & Value & $\begin{array}{c}\text { forest-agricultural } \\
\text { with built-up } \\
\text { areas }\end{array}$ & forest & forest-agricultural & $\begin{array}{c}\text { forest-agricultural } \\
\text { with built-up } \\
\text { areas }\end{array}$ \\
\hline & & & Score & 3 & 0 & 1 & 3 \\
\hline Mean score & & & & 1.7 & 1.4 & 1.6 & 1.9 \\
\hline Class of susceptibility & & & & III & II & III & III \\
\hline \multicolumn{2}{|c|}{ Characteristics of the catchment area } & & & $\begin{array}{l}\text { average } \\
\text { susceptibility to } \\
\text { load mobilisation } \\
\text { and moderate } \\
\text { capacity of load } \\
\text { supply }\end{array}$ & $\begin{array}{l}\text { low susceptibility } \\
\text { to load } \\
\text { mobilisation and } \\
\text { low capacity of } \\
\text { load supply }\end{array}$ & $\begin{array}{l}\text { average } \\
\text { susceptibility to } \\
\text { load mobilisation } \\
\text { and moderate } \\
\text { capacity of load } \\
\text { supply }\end{array}$ & $\begin{array}{l}\text { average } \\
\text { susceptibility to } \\
\text { load mobilisation } \\
\text { and moderate } \\
\text { capacity of load } \\
\text { supply }\end{array}$ \\
\hline
\end{tabular}

${ }^{1}$ - Filipiak and Raczyński (2000), ${ }^{2}$ - Czarnecka et al. (1989)

quirements of the Water Framework Directive (MGGP and IOŚ 2010; Bajkiewicz-Grabowska et al. 2011).

Annual phosphorus load to Strzeszowskie lake from area sources amounts to $168.3 \mathrm{~kg}\left(0.238 \mathrm{~g} \mathrm{~m}^{-2}\right)$, and nitrogen load - $5154.0 \mathrm{~kg}\left(7.290 \mathrm{~g} \mathrm{~m}^{-2}\right)$ (Table 7). In the direct catchment area, arable land is the main source of nutrient loading $(55.2 \%$ of nitrogen and $54.4 \%$ of phos- phorus), followed by surface run-off (39.5 and 31.7\% respectively). Atmospheric precipitation significantly contributes to the biogenic balance of the given lake. Annual atmospheric input amounts to $14.3 \%$ of phosphorus received from area sources and $17.7 \%$ of nitrogen load. The analysis of annual supply of real nutrient loading to Lake Strzeszowskie showed that waters supplying the

Table 3. Assessment of susceptibility to degradation according to Bajkiewicz-Grabowska (2002). Explanation: V - lake volume, SL - shoreline length, $\mathrm{ABA}$ - active bottom area, EV - epilimnion volume

\begin{tabular}{|c|c|c|c|c|c|c|c|c|c|c|c|c|c|c|c|c|}
\hline \multirow{3}{*}{ Lake } & \multirow{3}{*}{ Year } & \multicolumn{13}{|c|}{ Indices of susceptibility to degradation } & \multirow{2}{*}{\multicolumn{2}{|c|}{$\begin{array}{l}\text { Susceptibility to } \\
\text { degradation }\end{array}$}} \\
\hline & & \multicolumn{2}{|c|}{$\begin{array}{l}\text { Mean depth } \\
{[\mathrm{m}]}\end{array}$} & \multicolumn{2}{|c|}{$\begin{array}{c}\mathrm{V} / \mathrm{SL} \\
{\left[\mathrm{dm}^{3} \mathrm{~m}^{-1}\right]}\end{array}$} & \multicolumn{2}{|c|}{$\begin{array}{c}\text { Stratification } \\
{[\%]}\end{array}$} & \multicolumn{2}{|c|}{$\begin{array}{l}\mathrm{ABA} / \mathrm{EV} \\
{\left[\mathrm{m}^{2} \mathrm{~m}^{-3}\right]}\end{array}$} & \multicolumn{2}{|c|}{$\begin{array}{c}\text { Flushing } \\
\text { time } \\
{\left[\mathrm{yr}^{-1}\right]}\end{array}$} & \multicolumn{2}{|c|}{$\begin{array}{c}\text { Schindler's } \\
\text { ratio } \\
{\left[\mathrm{m}^{2} \mathrm{~m}^{-3}\right]}\end{array}$} & \multirow[t]{2}{*}{$\begin{array}{l}\text { Mean } \\
\text { score }\end{array}$} & & \\
\hline & & Value & Score & Value & Score & Value & Score & Value & Score & Value & Score & Value & Score & & Class & Resistance \\
\hline \multirow{3}{*}{$\begin{array}{l}\text { Strze- } \\
\text { szowskie }\end{array}$} & 2008 & \multirow{3}{*}{7.4} & \multirow{3}{*}{1} & \multirow{3}{*}{1.6} & \multirow{3}{*}{2} & 16.6 & 2 & 0.040 & 0 & \multirow{3}{*}{0.31} & \multirow{3}{*}{3} & \multirow{3}{*}{1.32} & \multirow{3}{*}{0} & 1.3 & II & medium \\
\hline & 2009 & & & & & 16.6 & 2 & 0.040 & 0 & & & & & 1.3 & II & medium \\
\hline & 2013 & & & & & 18.6 & 2 & 0.038 & 0 & & & & & 1.3 & II & medium \\
\hline \multirow[t]{3}{*}{ Dołgie } & 2008 & \multirow{3}{*}{3.1} & & \multirow{3}{*}{0.4} & \multirow{3}{*}{3} & 9.6 & 3 & 0.289 & 2 & \multirow{3}{*}{3.43} & \multirow{3}{*}{2} & \multirow{3}{*}{31.8} & \multirow{3}{*}{2} & 2.3 & III & low \\
\hline & 2009 & & & & & 0.0 & 3 & 0.319 & 3 & & & & & 2.5 & IV & nonresistant \\
\hline & 2013 & & & & & 8.8 & 3 & 0.305 & 3 & & & & & 2.5 & IV & nonresistant \\
\hline \multirow{3}{*}{$\begin{array}{l}\text { Swob- } \\
\text { nica }\end{array}$} & 2008 & \multirow{3}{*}{4.2} & \multirow{3}{*}{2} & \multirow{3}{*}{1.6} & & 0.0 & 3 & 0.237 & 2 & & & & & 2.0 & III & low \\
\hline & 2009 & & & & 2 & 0.0 & 3 & 0.237 & 2 & 0.84 & 3 & 7.35 & 0 & 2.0 & III & low \\
\hline & 2013 & & & & & 0.0 & 3 & 0.237 & 2 & & & & & 2.0 & III & low \\
\hline Dłużec & 2008 & & & & & 15.0 & 2 & 0.180 & 2 & & & & & 1.8 & III & low \\
\hline & 2009 & 6.1 & 1 & 0.9 & 3 & 15.0 & 2 & 0.180 & 2 & 3.30 & 2 & 23.7 & 1 & 1.8 & III & low \\
\hline & 2013 & & & & & 18.6 & 2 & 0.176 & 2 & & & & & 1.8 & III & low \\
\hline
\end{tabular}


Table 4. Assessment of susceptibility to degradation according to Kudelska et al. 1994. Explanation: V - lake volume, SL - shoreline length, $\mathrm{ABA}$ - active bottom area, EV - epilimnion volume

\begin{tabular}{|c|c|c|c|c|c|c|c|c|c|c|c|c|c|c|c|c|c|}
\hline \multirow{3}{*}{ Lake } & \multirow{3}{*}{ Year } & \multicolumn{14}{|c|}{ Indices of susceptibility to degradation } & & \\
\hline & & \multicolumn{2}{|c|}{$\begin{array}{c}\text { Mean } \\
\text { depth } \\
{[\mathrm{m}]}\end{array}$} & \multicolumn{2}{|c|}{$\begin{array}{c}\mathrm{V} / \mathrm{SL} \\
{\left[\mathrm{dm}^{3} \mathrm{~m}^{-1}\right]}\end{array}$} & \multicolumn{2}{|c|}{$\begin{array}{c}\text { Stratification } \\
{[\%]}\end{array}$} & \multicolumn{2}{|c|}{$\begin{array}{c}\mathrm{ABA} / \mathrm{EV} \\
{\left[\mathrm{m}^{2} \mathrm{~m}^{-3}\right]}\end{array}$} & \multicolumn{2}{|c|}{$\begin{array}{c}\text { Water ex- } \\
\text { change } \\
{\left[\% \mathrm{yr}^{-1}\right]}\end{array}$} & \multicolumn{2}{|c|}{$\begin{array}{c}\text { Schindler's } \\
\text { ratio } \\
{\left[\mathrm{m}^{2} \mathrm{~m}^{-3}\right]}\end{array}$} & \multirow{2}{*}{$\begin{array}{c}\begin{array}{c}\text { Land } \\
\text { use }\end{array} \\
\text { Score }\end{array}$} & \multirow{2}{*}{$\begin{array}{r}\text { Mean } \\
\text { - score }\end{array}$} & \multicolumn{2}{|c|}{$\begin{array}{c}\text { Susceptibility to } \\
\text { degradation }\end{array}$} \\
\hline & & Value & Score & Value & Score & Value & Score & Value & Score & Value & Score & Value & Score & & & Class & $\begin{array}{l}\text { Resis- } \\
\text { tance }\end{array}$ \\
\hline \multirow{3}{*}{$\begin{array}{l}\text { Strze- } \\
\text { szowskie }\end{array}$} & 2008 & \multirow{3}{*}{7.4} & \multirow{3}{*}{2} & \multirow{3}{*}{1.6} & \multirow{3}{*}{3} & 16.6 & 3 & 0.040 & 1 & \multirow{3}{*}{31.8} & \multirow{3}{*}{2} & \multirow{3}{*}{1.32} & \multirow{3}{*}{1} & 3 & 2.14 & II & medium \\
\hline & 2009 & & & & & 16.6 & 3 & 0.040 & 1 & & & & & 3 & 2.14 & II & medium \\
\hline & 2013 & & & & & 18.6 & 3 & 0.038 & 1 & & & & & 3 & 2.14 & II & medium \\
\hline \multirow[t]{3}{*}{ Dołgie } & 2008 & \multirow{3}{*}{3.1} & \multirow{3}{*}{3} & \multirow{3}{*}{0.4} & \multirow{3}{*}{4} & 9.6 & 4 & 0.289 & 3 & \multirow{3}{*}{343.6} & \multirow{3}{*}{3} & \multirow{3}{*}{31.8} & \multirow{3}{*}{3} & 3 & 3.29 & IV & $\begin{array}{l}\text { nonre- } \\
\text { sistant }\end{array}$ \\
\hline & 2009 & & & & & 0.0 & 4 & 0.319 & 4 & & & & & & 3.43 & IV & $\begin{array}{l}\text { nonre- } \\
\text { sistant }\end{array}$ \\
\hline & 2013 & & & & & 8.8 & 4 & 0.305 & 4 & & & & & & 3.43 & IV & $\begin{array}{l}\text { nonre- } \\
\text { sistant }\end{array}$ \\
\hline \multirow{3}{*}{$\begin{array}{l}\text { Swob- } \\
\text { nica }\end{array}$} & 2008 & \multirow{3}{*}{4.2} & \multirow{3}{*}{3} & & & 0.0 & 4 & 0.237 & 3 & & & & & 2 & 2.71 & III & low \\
\hline & 2009 & & & 1.6 & 3 & 0.0 & 4 & 0.237 & 3 & 83.5 & 2 & 7.35 & 2 & & 2.71 & III & low \\
\hline & 2013 & & & & & 0 & 4 & 0.237 & 3 & & & & & & 2.71 & III & low \\
\hline Dłużec & 2008 & & & & & 15.0 & 3 & 0.180 & 3 & & & & & 3 & 2.86 & III & low \\
\hline & 2009 & 6.1 & 2 & 0.9 & 3 & 15.0 & 3 & 0.180 & 3 & 330.5 & 3 & 23.70 & 3 & & 2.86 & III & low \\
\hline & 2013 & & & & & 18.6 & 3 & 0.176 & 3 & & & & & & 2.86 & III & low \\
\hline
\end{tabular}

Direct catchment land use categories: $\geq 60 \%$ of forest $-1,<60 \%$ of forest and $<60 \%$ of arable land $-2, \geq 60 \%$ of arable land -3 , catchment with urban development -4

Table 5. Catchment-lake ecological system type and eutrophication rate of the studied lakes (Bajkiewicz-Grabowska 2002)

\begin{tabular}{|c|c|c|c|c|c|}
\hline Lake & $\begin{array}{l}\text { Group of the catchment } \\
\text { capacity to mobilise the load }\end{array}$ & $\begin{array}{c}\text { Class of the susceptibility } \\
\text { to degradation }\end{array}$ & \multicolumn{2}{|c|}{ Eutrophication rate } & Catchment-lake system \\
\hline Strzeszowskie & 3 & II & \multirow[t]{2}{*}{ Moderate } & \multirow[t]{2}{*}{ Type 2} & \multirow{2}{*}{$\begin{array}{l}\text { High lake resistance, unfavourable } \\
\text { catchment conditions, high possibility of } \\
\text { mobilisation of area load, moderate rate } \\
\text { of eutrophication }\end{array}$} \\
\hline Dłużec & 3 & II & & & \\
\hline Dołgie & 3 & III, IV & \multirow[t]{2}{*}{ Rapid } & \multirow[t]{2}{*}{ Type 4} & \multirow{2}{*}{$\begin{array}{l}\text { Natural features of the catchment } \\
\text { promote surface run-offs, lake is highly } \\
\text { susceptible to external pressure, rapid } \\
\text { eutrophication of the lake }\end{array}$} \\
\hline Swobnica & 3 & III & & & \\
\hline
\end{tabular}

Table 6. Land use type in direct catchment of the analysed lakes (EEA 2006)

\begin{tabular}{|c|c|c|c|c|c|c|c|c|}
\hline Lake & Land use & Unit & Forests & Grasslands & Waters & Arable land & Other & Total \\
\hline \multirow[t]{4}{*}{ Strzeszowskie } & \multirow[t]{2}{*}{ Direct catchment } & {$\left[\mathrm{km}^{2}\right]$} & 0.1 & 0.5 & 1.3 & 2.6 & 0.5 & 5.0 \\
\hline & & [\%] & 2.2 & 10.4 & 25.6 & 52.6 & 9.2 & 100 \\
\hline & \multirow[t]{2}{*}{ Total catchment } & {$\left[\mathrm{km}^{2}\right]$} & 1.8 & 0.5 & 1.4 & 6.8 & 0.7 & 11.2 \\
\hline & & {$[\%]$} & 15.7 & 4.6 & 12.7 & 60.8 & 6.2 & 100 \\
\hline \multirow[t]{4}{*}{ Dołgie } & \multirow[t]{2}{*}{ Direct catchment } & {$\left[\mathrm{km}^{2}\right]$} & 4.7 & 0.2 & 0.6 & 2.4 & $>0.1$ & 7.9 \\
\hline & & {$[\%]$} & 59.5 & 2 & 7.6 & 29.9 & 1.0 & 100 \\
\hline & \multirow[t]{2}{*}{ Total catchment } & {$\left[\mathrm{km}^{2}\right]$} & 12.9 & 4.6 & 0.9 & 36.1 & 1 & 55.4 \\
\hline & & {$[\%]$} & 23.2 & 8.3 & 1.6 & 65.2 & 1.7 & 100 \\
\hline \multirow[t]{4}{*}{ Swobnica } & \multirow[t]{2}{*}{ Direct catchment } & {$\left[\mathrm{km}^{2}\right]$} & 3.9 & - & 3.4 & 3.8 & 0.3 & 11.4 \\
\hline & & {$[\%]$} & 34.1 & - & 30.4 & 33.3 & 2.2 & 100 \\
\hline & \multirow[t]{2}{*}{ Total catchment } & {$\left[\mathrm{km}^{2}\right]$} & 26.8 & 5.4 & 5.1 & 62.7 & 2.9 & 102.9 \\
\hline & & {$[\%]$} & 26.0 & 5.3 & 5.0 & 60.9 & 2.8 & 100 \\
\hline \multirow[t]{4}{*}{ Dłużec } & \multirow[t]{2}{*}{ Direct catchment } & {$\left[\mathrm{km}^{2}\right]$} & 0.5 & 0.4 & 1.0 & 9.5 & 0.6 & 12.0 \\
\hline & & {$[\%]$} & 4.3 & 3.2 & 7.9 & 79.5 & 5.1 & 100 \\
\hline & \multirow[t]{2}{*}{ Total catchment } & {$\left[\mathrm{km}^{2}\right]$} & 28.4 & 7.0 & 6.1 & 79.1 & 4.1 & 124.7 \\
\hline & & {$[\%]$} & 22.8 & 5.6 & 4.9 & 63.4 & 3.3 & 100 \\
\hline
\end{tabular}


Table 7. Phosphorus and nitrogen load from catchment

\begin{tabular}{|c|c|c|c|c|c|c|c|c|c|}
\hline \multirow{2}{*}{ Type of load } & \multirow{2}{*}{ Unit $P$} & \multicolumn{2}{|c|}{ Dłużec } & \multicolumn{2}{|c|}{ Swobnica } & \multicolumn{2}{|c|}{ Dołgie } & \multicolumn{2}{|c|}{ Strzeszowskie } \\
\hline & & $\mathrm{N}$ & $\mathrm{P}$ & $\mathrm{N}$ & $\mathrm{P}$ & $\mathrm{N}$ & $\mathrm{P}$ & $\mathrm{N}$ & $\mathrm{P}$ \\
\hline Load from direct catchment & {$\left[\mathrm{kg} \mathrm{yr}^{-1}\right]$} & 240.6 & 6618.2 & 146.2 & 3806.1 & 144.0 & 3699.0 & 143.5 & 4242.0 \\
\hline arable land & [\%] & 86.6 & 94.4 & 70.7 & 81.5 & 50.0 & 58.4 & 54.4 & 55,2 \\
\hline grassland & [\%] & 2.8 & 1.5 & 0.7 & 0.4 & 2.8 & 1.6 & 7.0 & 3,5 \\
\hline forests & [\%] & 1.7 & 0.9 & 24.6 & 14.2 & 32.6 & 19.1 & 0.7 & 0,4 \\
\hline built-in areas & [\%] & 8.0 & 1.9 & 1.5 & 0.4 & - & - & 6.3 & 1,4 \\
\hline waters & [\%] & 0.9 & 1.3 & 2.5 & 3.5 & 14.6 & 20.9 & 31.7 & 39,5 \\
\hline Atmospheric input & {$\left[\mathrm{kg} \mathrm{yr}^{-1}\right]$} & 24.8 & 912.0 & 121.5 & 4478.9 & 24.8 & 912.0 & 24.8 & 912.0 \\
\hline Load from areal sources & {$\left[\mathrm{kg} \mathrm{yr}^{-1}\right]$} & 265.4 & 7530.2 & 267.7 & 8285.0 & 168.8 & 4611.0 & 168.3 & 5154.0 \\
\hline Tributaries & [\%] & 6627.2 & 32369.7 & 4345.0 & 22953.3 & 1936.1 & 8443.4 & 1570.5 & 7044,5 \\
\hline Total load & {$\left[\mathrm{kg} \mathrm{yr}^{-1}\right]$} & 6892.6 & 39899.8 & 4612.7 & 31238.3 & 2104.9 & 13054.4 & 1738.5 & 12198.5 \\
\hline
\end{tabular}

Table 8. Anthropogenic pressure on Lake Strzeszowskie

\begin{tabular}{|c|c|c|c|c|}
\hline Real phosphorus load (RPL) & Permissible phosphorus load (PPL) ${ }^{1}$ & Dangerous phosphorus load (DPL) ${ }^{1}$ & \multirow{2}{*}{ RPL : PPL } & \multirow{2}{*}{ RPL : DPL } \\
\hline$\left[\mathrm{g} \mathrm{m}^{-2} \mathrm{yr}^{-1}\right]$ & {$\left[\mathrm{g} \mathrm{m}^{-2} \mathrm{yr}^{-1}\right]$} & {$\left[\mathrm{g} \mathrm{m}^{-2} \mathrm{yr}^{-1}\right]$} & & \\
\hline 0.238 & 0.089 & 0.178 & 2.7 & 1.3 \\
\hline Real nitrogen load (RNL) & Permissible nitrogen load (PNL) ${ }^{1}$ & 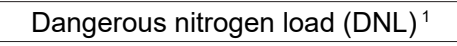 & \multirow{2}{*}{ RNL : PNL } & \multirow{2}{*}{ RNL : DNL } \\
\hline$\left[\mathrm{g} \mathrm{m}^{-2} \mathrm{yr}^{-1}\right]$ & {$\left[\mathrm{g} \mathrm{m}^{-2} \mathrm{yr}^{-1}\right]$} & {$\left[\mathrm{g} \mathrm{m}^{-2} \mathrm{yr}^{-1}\right]$} & & \\
\hline 12.828 & 1.336 & 2.672 & 9.6 & 4.8 \\
\hline
\end{tabular}

$\mathrm{RPL}>\mathrm{DPL}$ and RNL > DNL

Lake susceptibility class III

High probability of rapid progression of eutrophication

laccording to Vollenweider (1968) model

reservoirs are the main source of biogenic compounds (90.3\% of phosphorus and $57.7 \%$ of nitrogen). It was found that the real load of nitrogen and phosphorus was higher than values of permissible loading (trophic state unchanged) as well as excessive loading (encouraging phytoplankton growth) (Table 8). Strzeszowskie lake can be classified as class III in terms of the threat of eutrophication i.e., there is a high risk of a rapid progression of eutrophication for this lake (Table 8).

Lake Dołgie is also characterised by the fact that the fundamental share of phosphorus and nitrogen load from the direct catchment area was supplied by agricultural areas (50.0 and 58.4\%), followed by forested areas, surface run-off and atmospheric precipitation (Tables 7 and 9). The predominant share of total phosphorus $(92.0 \%)$ and nitrogen (64.7\%) load was supplied to the lake by the River Tywa (Table 9). However, it must be ex- plicitly stated that in the case of this particular lake, levels of both biogenic elements significantly exceeded the permissible and excessive levels (Tables 7 and 9). Therefore, Lake Dołgie was classified as class III - high probability of intensification of eutrophication processes.

On the grounds of the obtained results, Lake Dłużec was also classified as class III in terms of risk of eutrophication. The real load of biogenic compounds was higher than the permissible as well as excessive level both for phosphorus and nitrogen (Tables 7 and 10). In the case of Lake Dłużec, arable land was a considerable source of biogenic supply from the direct catchment area, significantly less was delivered by surface run-off followed by urban areas. However, the River Tywa constituted the main source of biogenic supply - 96.2 and $81.1 \%$ of the total nutrient loading supplied to the lake (Tables 7 and 10).

Table 9. Anthropogenic pressure on Lake Dołgie

\begin{tabular}{|c|c|c|c|c|}
\hline Real phosphorus load (RPL) & Permissible phosphorus load (PPL) ${ }^{1}$ & Dangerous phosphorus load (DPL) ${ }^{1}$ & \multirow{2}{*}{ RPL : PPL } & \multirow{2}{*}{ RPL : DPL } \\
\hline$\left[\mathrm{g} \mathrm{m}^{-2} \mathrm{yr}^{-1}\right]$ & {$\left[\mathrm{g} \mathrm{m}^{-2} \mathrm{yr}^{-1}\right]$} & {$\left[\mathrm{g} \mathrm{m}^{-2} \mathrm{yr}^{-1}\right]$} & & \\
\hline 3.678 & 0.170 & 0.340 & 14.2 & 7.1 \\
\hline Real nitrogen load (RNL) & Permissible nitrogen load (PNL) ${ }^{1}$ & Dangerous nitrogen load (DNL) ${ }^{1}$ & \multirow{2}{*}{ RNL : PNL } & \multirow{2}{*}{ RNL : DNL } \\
\hline$\left[\mathrm{g} \mathrm{m}^{-2} \mathrm{yr}^{-1}\right]$ & {$\left[\mathrm{g} \mathrm{m}^{-2} \mathrm{yr}^{-1}\right]$} & {$\left[\mathrm{g} \mathrm{m}^{-2} \mathrm{yr}^{-1}\right]$} & & \\
\hline 21.519 & 2.547 & 5.093 & 8.4 & 4.2 \\
\hline \multicolumn{5}{|l|}{$\mathrm{RPL}>\mathrm{DPL}$ and $\mathrm{RNL}>\mathrm{DNL}$} \\
\hline \multicolumn{5}{|c|}{ Lake susceptibility class III } \\
\hline High probability of rapid progre & sion of eutrophication & & & \\
\hline
\end{tabular}


Table 10. Anthropogenic pressure on Lake Dłużec

\begin{tabular}{|c|c|c|c|c|}
\hline Real phosphorus load (RPL) & Permissible phosphorus load (PPL) ${ }^{1}$ & Dangerous phosphorus load (DPL) $^{1}$ & \multirow{2}{*}{ RPL : PPL } & \multirow{2}{*}{ RPL : DPL } \\
\hline$\left[\mathrm{g} \mathrm{m}^{-2} \mathrm{yr}^{-1}\right]$ & {$\left[\mathrm{g} \mathrm{m}^{-2} \mathrm{yr}^{-1}\right]$} & {$\left[\mathrm{g} \mathrm{m}^{-2} \mathrm{yr}^{-1}\right]$} & & \\
\hline 8.154 & 0.344 & 0.688 & 23.70 & 11.85 \\
\hline Real nitrogen load (RNL) & Permissible nitrogen load (PNL) ${ }^{1}$ & Dangerous nitrogen load (DNL) ${ }^{1}$ & \multirow{2}{*}{ RNL : PNL } & \multirow{2}{*}{ RNL : DNL } \\
\hline$\left[\mathrm{g} \mathrm{m}^{-2} \mathrm{yr}^{-1}\right]$ & {$\left[\mathrm{g} \mathrm{m}^{-2} \mathrm{yr}^{-1}\right]$} & {$\left[\mathrm{g} \mathrm{m}^{-2} \mathrm{yr}^{-1}\right]$} & & \\
\hline 48.643 & 5.157 & 10.314 & 9.43 & 4.72 \\
\hline
\end{tabular}

Lake susceptibility class III

High probability of rapid progression of eutrophication

'according to Vollenweider (1968) model

Table 11. Anthropogenic pressure on Lake Swobnica

\begin{tabular}{|c|c|c|c|c|}
\hline Real phosphorus load (RPL) & Permissible phosphorus load (PPL) ${ }^{1}$ & Dangerous phosphorus load (DPL) ${ }^{1}$ & \multirow{2}{*}{ RPL : PPL } & \multirow{2}{*}{ RPL : DPL } \\
\hline$\left[\mathrm{g} \mathrm{m}^{-2} \mathrm{yr}^{-1}\right]$ & {$\left[\mathrm{g} \mathrm{m}^{-2} \mathrm{yr}^{-1}\right]$} & {$\left[\mathrm{g} \mathrm{m}^{-2} \mathrm{yr}^{-1}\right]$} & & \\
\hline 1.340 & 0.058 & 0.116 & 23.1 & 11.6 \\
\hline Real nitrogen load (RNL) & Permissible nitrogen load (PNL) ${ }^{1}$ & Dangerous nitrogen load (DNL) ${ }^{1}$ & \multirow{2}{*}{ RNL : PNL } & \multirow{2}{*}{ RNL : DNL } \\
\hline$\left[\mathrm{g} \mathrm{m}^{-2} \mathrm{yr}^{-1}\right]$ & {$\left[\mathrm{g} \mathrm{m}^{-2} \mathrm{yr}^{-1}\right]$} & {$\left[\mathrm{g} \mathrm{m}^{-2} \mathrm{yr}^{-1}\right]$} & & \\
\hline 9.059 & 0.870 & 1.740 & 10.4 & 5.2 \\
\hline \multicolumn{5}{|l|}{$\mathrm{RPL}>\mathrm{DPL}$ and $\mathrm{RNL}>\mathrm{DNL}$} \\
\hline \multicolumn{5}{|l|}{ Lake susceptibility class III } \\
\hline High probability of rapid progre & sion of eutrophication & & & \\
\hline
\end{tabular}

'according to Vollenweider (1968) model

Annual phosphorus load to Lake Swobnica amounted to $4612.71 \mathrm{~kg}$, and nitrogen $31238.2 \mathrm{~kg}$, out of which a significant share was supplied by the River Tywa $94.7 \%$ of phosphorus and $73.5 \%$ of nitrogen load. Nutrient loading supply from area sources to Lake Swobnica was insignificant and the most prominent sources were run-offs from agricultural land and forests. In the case of this reservoir, similarly to the three remaining lakes under analysis, real nutrient loading was significantly higher than permissible and excessive load levels. Therefore, the lake can be classified as class III in terms of risk of eutrophication, which explicitly suggests the high probability of a rapid progression of the process (Tables 7 and 11).

Considerably high phosphorus and nitrogen content in the waters of the lakes under analysis confirmed a high level of eutrophication. The reservoirs are highly eutrophic and the biogenic content indicates a risk of further intensification of the eutrophication process.

Anthropogenic pressure as well as the influence of the catchment area greatly affect the quality of water in the reservoirs under analysis. The values of indices discussed below unambiguously confirm the high level of eutrophication of the reservoirs in question (Table 12).

Total phosphorus and phosphate content in surface waters in spring, and in bottom waters in summer, clearly demonstrated a high rate of eutrophication. This was confirmed by the mineral nitrogen content and conductivity values obtained in the course of the analysis. The analysed lakes are characterised by an increased content of organic matter in summer (termed seston dry mass and chlorophyll $a$ ) especially in surface water, and markedly lower content in bottom waters, particularly in the deepest lake - Strzeszowskie. Content of organic matter was higher in lakes located in the downstream part of the River Tywa. Substantial content of organic matter results in unfavourable optical conditions of the analysed lakes, particularly in Dołgie and Swobnica lakes. However, oxygen conditions were found to be favourable. In polymictic Lake Swobnica as well as in the hypolimnion water in stratified lakes, deoxygenation was not found (Table 12).

Determination of natural susceptibility to degradation and the influence of catchment area, and particularly identification of nutrient loading supplied to the lakes from numerous sources as well as water quality in the given area provide grounds for distinguishing protective action to be taken for individual reservoirs.

The assumptions mainly focus on outlining the action to be taken regarding catchment area, methods of lake protection (together with adequate fishery economy) and establishing methods for the remediation of the reservoirs (Dąbrowska-Prot and Hillbricht-Ilkowska 1991).

Since the main source of nutrient loading is surface run-off and the catchment area is dominated by agricultural production, protective action should be conducted by means of introducing good agricultural practice in this area. As a general rule, the following should be introduced: limitation of the amount of pollutants from the point sources (necessary inventorying and balancing) and limitation of area sources in agro-ecosystems, 
Table 12. Assessment of water quality of the analysed lakes (according to Kudelska et al. 1994)

\begin{tabular}{|c|c|c|c|c|c|c|c|c|c|c|c|c|c|c|c|}
\hline \multirow{3}{*}{ Parameter } & \multirow{3}{*}{ Unit } & \multirow{3}{*}{ Season } & \multirow{3}{*}{ Layer } & \multicolumn{12}{|c|}{ Lake / year } \\
\hline & & & & \multicolumn{3}{|c|}{ Strzeszowskie } & \multicolumn{3}{|c|}{ Dołgie } & \multicolumn{3}{|c|}{ Swobnica } & \multicolumn{3}{|c|}{ Dłużec } \\
\hline & & & & 2008 & 2009 & 2013 & 2008 & 2009 & 2013 & 2008 & 2009 & 2013 & 2008 & 2009 & 2013 \\
\hline $\mathrm{MHO}$ & {$[\%]$} & summer & bottom & 48.0 & 49.2 & 50.70 & 69.4 & 69.6 & 65.2 & \multicolumn{3}{|c|}{ polymictic } & 78.5 & 29.4 & 33.0 \\
\hline $\mathrm{O}_{2}$ content & {$\left[\mathrm{mg} \mathrm{dm}^{-3}\right]$} & summer & bottom & - & - & - & - & - & - & 9.1 & 9.1 & 9.3 & - & - & - \\
\hline $\mathrm{COD}_{\mathrm{CR}}$ & {$\left[\mathrm{mg} \mathrm{dm}^{-3}\right]$} & summer & surface & 30.6 & 29.2 & 28.4 & 29.6 & 32.9 & 32.0 & 25.6 & 21.6 & 22.4 & 30.6 & 31.2 & 28.6 \\
\hline $\mathrm{BOD}_{5}$ & {$\left[\mathrm{mg} \mathrm{dm}^{-3}\right]$} & summer & surface & 4.5 & 4.9 & 4.6 & 4.8 & 4.2 & 4.2 & 6.2 & 5.4 & 4.8 & 6.4 & 4.8 & 4.6 \\
\hline $\mathrm{BOD}_{5}$ & {$\left[\mathrm{mg} \mathrm{dm}^{-3}\right]$} & summer & bottom & 1.8 & 2.0 & 2.0 & 4.0 & 4.2 & 4.1 & - & - & - & 4.4 & 3.4 & 3.6 \\
\hline $\mathrm{P}_{-} \mathrm{PO}_{4}$ & {$\left[\mathrm{mg} \mathrm{dm}^{-3}\right]$} & spring & surface & 0.040 & 0.060 & 0.055 & 0.040 & 0.043 & 0.045 & 0.050 & 0.070 & 0.065 & 0.050 & 0.070 & 0.066 \\
\hline $\mathrm{P}_{-} \mathrm{PO}_{4}$ & {$\left[\mathrm{mg} \mathrm{dm}^{-3}\right]$} & summer & bottom & 0.120 & 0.150 & 0.140 & 0.150 & 0.165 & 0.155 & - & - & - & 0.090 & 0.090 & 0.088 \\
\hline TP & {$\left[\mathrm{mg} \mathrm{dm}^{-3}\right]$} & summer & bottom & 0.230 & 0.280 & 0.222 & 0.220 & 0.212 & 0.245 & - & - & - & 0.340 & 0.260 & 0.288 \\
\hline TP & {$\left[\mathrm{mg} \mathrm{dm}^{-3}\right]$} & $\begin{array}{c}\text { mean } \\
(\mathrm{spg}+\mathrm{sur})\end{array}$ & surface & 0.310 & 0.315 & 0.308 & 0.220 & 0.295 & 0.300 & 0.335 & 0.330 & 0.325 & 0.230 & 0.200 & 0.225 \\
\hline N-mineral & {$\left[\mathrm{mg} \mathrm{dm}^{-3}\right]$} & summer & surface & 0.57 & 0.56 & 0.55 & 1.05 & 0.95 & 1.05 & 0.80 & 0.59 & 0.82 & 0.66 & 0.84 & 0.88 \\
\hline $\mathrm{N}-\mathrm{NH}_{4}$ & {$\left[\mathrm{mg} \mathrm{dm}^{-3}\right]$} & summer & bottom & 0.322 & 0.372 & 0.333 & 0.333 & 0.289 & 0.301 & - & - & - & 0.048 & 0.372 & 0.236 \\
\hline TN & {$\left[\mathrm{mg} \mathrm{dm}^{-3}\right]$} & $\begin{array}{c}\text { mean } \\
\text { (spg+sur) }\end{array}$ & surface & 1.15 & 1.38 & 1.28 & 1.59 & 1.63 & 1.65 & 1.57 & 1.43 & 1.65 & 1.23 & 1.59 & 1.62 \\
\hline Cond. $\left(20^{\circ} \mathrm{C}\right)$ & {$\left[\mu \mathrm{S} \mathrm{cm}^{-1}\right]$} & spring & surface & 519 & 503 & 498 & 523 & 558 & 533 & 489 & 488 & 472 & 488 & 481 & 479 \\
\hline Chlorophyll-a & {$\left[\mathrm{mg} \mathrm{m}^{-3}\right]$} & $\begin{array}{c}\text { mean } \\
\text { (spg+sur) }\end{array}$ & surface & 18.6 & 22.9 & 19.5 & 16.8 & 19.6 & 18.5 & 15.4 & 21.1 & 17.5 & 14.4 & 15.7 & 16.2 \\
\hline Seston d.m. & {$\left[\mathrm{mg} \mathrm{dm}^{-3}\right]$} & $\begin{array}{c}\text { mean } \\
\text { (spg+sur) }\end{array}$ & surface & 17.3 & 16.5 & 16.0 & 16.9 & 13.0 & 14.0 & 21.5 & 18.0 & 18.0 & 17.8 & 16.3 & 17.0 \\
\hline Secchi disc & {$[\mathrm{m}]$} & $\begin{array}{c}\text { mean } \\
\text { (spg+sur) }\end{array}$ & surface & 2.0 & 2.8 & 2.3 & 1.4 & 1.6 & 1.6 & 1.3 & 1.5 & 1.3 & 1.3 & 2.0 & 2.0 \\
\hline
\end{tabular}

MHO - mean hypolimnetic oxigenation

agricultural landscape and water/land contact zones (Bechmann et al. 2005; Ptak et al. 2013).

The analysed lakes are characterised by unfavourable natural conditions in terms of eutrophication. The natural features of the catchment areas foster surface run-off, and the reservoirs are highly susceptible to external influences which results in a high rate of lake eutrophication. Poor water quality mainly results from high natural vulnerability to anthropogenic pressure (Table 7). Action aimed at improving the state of the lakes under analysis should be connected with improving the quality of water in the River Tywa and changing the land management within the catchment area of the lakes.

Action designed to improve the ecosystem status of Lake Strzeszowskie should be centred on a substantial change of catchment area use by eliminating the sources of pollution. Additionally, remediation of the reservoir is recommended. Improving the state of the remaining three lakes requires long-term comprehensive action within their catchment areas aimed at eliminating the sources of external loading. In addition, it is necessary to implement measures designed to reduce the "internal" stagnation of lakes by means of adequate remediation techniques.

Due to the importance of the water quality of the River Tywa for the ecological status of the analysed lakes, action aimed at regulating wastewater management should be taken in the area of the entire catch- ment. In order to reduce nutrient loading in waters of the River Tywa, is it mandatory to enforce compliance with good agricultural practice in agricultural areas within the catchment area, especially within the direct catchment area.

With respect to fertilisation and storage of fertilisers within the catchment area, it is advisable to promote the use of natural fertilisers in the agricultural area in amounts not greater than $170 \mathrm{~kg} \mathrm{~N} \mathrm{ha}^{-1}$ per year. Manure fertilisation should be used only in early spring, and application of manure to fields in late autumn should be allowed providing that the fertiliser is immediately ploughed into the soil. Slurry fertilisation in the catchment area and the use of wastewater and sludge in arable land within the direct catchment area ought to be prohibited.

Nitrogen mineral fertilisation should be applied immediately before the period of maximum nutrient demand in plants. Moreover, in the strip of $50 \mathrm{~m}$ width from the lake, solid mineral fertilisers should be applied manually.

In terms of livestock farming the following should be prohibited: grazing in periods of excessive soil moisture and after the first half of October, herd grazing of livestock within a $100 \mathrm{~m}$ area from the lake shore, watering livestock directly from the lake and locating new farming plants or non-bedding stock farming within the catchment area. Livestock farms located within an area of $100 \mathrm{~m}$ from the lake shore ought to be equipped 
with an impermeable manure storage structure and an adequate leachate drainage system with hermetic tanks. Green fodder is to be stored on an insulated surface to prevent silage juice from reaching the water or soil.

Protective action taken in terms of plant production should include prohibition of cultivation in the direct catchment area parallel to the lake shore, and an obligatory practice of alternating crops.

In order to prevent the release of biogenic compounds from the direct catchment to soil, the shore zone should be especially protected by preventing the devastation of natural vegetation and widening the vegetation strips (particularly on the arable land side) to a minimum of $15 \mathrm{~m}$ from the shoreline. Tree or bush strips of approximately $15 \mathrm{~m}$ in width should be established on sections of shoreline where such isolation is missing.

Angling in the area of the analysed lakes should be limited, this should particularly apply to lure fishing (Wołos and Mioduszewska 2003). Detailed limitations to lake use and guidelines concerning protection are presented in the study (MGGP and IOS 2010).

\section{Conclusions}

The analysis of lakes Strzeszowskie, Dołgie, Swobnica and Dłużec provides a basis for forming an opinion on the ecological status of the reservoirs and allows directions for action aimed at reducing or reversing the negative effects of excessive eutrophication to be delineated.

The assessment of catchment in terms of mobilisation and supply of biogenic matter to lakes ranks the catchment areas of the analysed lakes as III class catchment with moderate possibility of matter supply to lakes. Swobnica and Dłużec lakes are characterised by low resistance to degradation. Lake Strzeszowskie is categorised as having moderate resistance and Lake Dołgie is non-resistant to degradation and highly susceptible to external pressure. The rate of eutrophication of lakes Strzeszowskie and Dłużec was found to be moderate, whereas lakes Dołgie and Swobnica show a high rate of eutrophication.

The analysis of nutrient loading reaching the waters of the aforementioned lakes shows that the main area source is arable land, and the fundamental point source is the inflow or river waters to each of the analysed reservoirs. Nutrient loading supplied by the river is several times higher than area load. Such conditions require protective action to be taken within the catchment area and, particularly, intensive implementation of good agricultural practice.

The marked predominance of real nutrient loading supplied to the lakes over permissible and excessive load values (e.g. excess over nitrogen permissible load values in Lake Dłużec by $2370 \%$, Swobnica - 2310\%, Dołgie - 1420\%, Strzeszowskie - 270\%) provides grounds for classification of the analysed lakes as III class of eutrophication threat with a high rate of the process.

\section{References}

Bajkiewicz-Grabowska E., 2002, Obieg materii w systemach rzeczno-jeziornych (Circulation of matter in the riverlake systems), Wydaw. UW, Wydz. Geogr. Stud. Regional., Warszawa, 272 pp (in Polish, English summary).

Bajkiewicz-Grabowska E., Kwidzyńska M., Grabowska B., Markowski M., Trusewicz Zb., 2011, Przegląd i analiza jezior wskazanych do derogacji w obszarze regionu wodnego Dolnej Odry i Pomorza Zachodniego (Review and analysis of lakes identified for derogation in the Lower Oder and West Pomerania) [typescript], RZGW Szczecin, Gdańsk, 400 pp (in Polish).

Bechmann M.E., Bergeb D., Eggestada H.O, Vandsemba S.M., 2005, Phosphorus transfer from agricultural areas and its impact on the eutrophication of lakes - two longterm integrated studies from Norway, J. Hydrol. 304(14): 238-250.

Borowiak M., Maślanka W., 2007, Wpływ wieloletniej antropopresji na środowisko abiotyczne Jeziora Klasztornego Małego (Impact of long-term anthropopression on the abiotic environment of the Lake Klasztorne Małe), [in:] Kostrzewski A., Szpikowski J. (eds), Funkcjonowanie geoekosystemów zlewni rzecznych. T. 4: Procesy ekstremalne w środowisku geograficznym (Functioning of river basin geoecosystems. Vol. 4: Extreme processes in a geographical environment), Bogucki Wydaw. Nauk., Poznań: 239-252 (in Polish).

Czarnecka H., Bialuk J., Hołdakowska J., Marcinkowska Z., Woroncow T., Majewska I., 1989, Ocena ilościowa i fizykochemiczna zasobów wodnych jezior województwa szczecińskiego. Cz. 4: Zlewnie jezior województwa szczecińskiego (Quantitative and phisicochemical estimation of water resources of the Szczecin Voivodeship lakes. Part 4: The catchment basins of the Szczecin Voivodeship lakes) [typescript], IMGW, Warszawa (in Polish).

Dąbrowska-Prot E., Hillbricht-Ilkowska A., 1991, Struktura i funkcjonowanie krajobrazu pojeziernego: próba ekologicznego spojrzenia, kierunki ochrony (The structure and functioning of a Lake District Landscape: An attempt at an ecological approach, guidelines for protection), Zesz. Nauk. Kom. Nauk “Człowiek i środowisko" PAN 15: 237249 (in Polish, English summary).

[EEA] European Environment Agency, 2006, Corine Land Cover. Environmental interactive maps [online]. Retrieved from http://www.eea.europa.eu/data-and-maps/ explore-interactive-maps\#c5 $=\& \mathrm{c} 0=0 \& \mathrm{~b} \_s t a r t=0 \quad[\mathrm{ac}-$ cessed 16 May 2017]. 
Filipiak J., Raczyński M., 2000, Jeziora Zachodniopomorskie. Zarys faktografii (Western Pomeranian lakes. A book of facts), Wydaw. AR Szczec., Szczecin, 255 pp (in Polish).

Filipiak J., Sadowski J., 1994, Jeziora szczecińskie. Zarys faktografii (Szczecin Lakes. A book of facts) Wydaw. AR Szczec., Szczecin, 260 pp (in Polish).

Górniak A., Więcko A., Karpowicz M., 2016, Changes in the trophic status of lakes in the Suwałki Landscape Park (NE Poland), Limnol. Rev. 16(4): 221-227.

Jarosiewicz A., Ficek D., Zapadka T., 2011, Eutrophication parameters and Carlson-type trophic state indices in selected Pomeranian lakes, Limnol. Rev. 11(1): 15-23.

Jonge V.N., Elliott M., Orive E., 2002, Causes, historical development, effects and future challenges of a common environmental problem: eutrophication, Hydrobiologia 475(1): 1-19.

Kato T., Kuroda H., Nakasone H., 2009, Runoff characteristics of nutrients from an agricultural watershed with intensive livestock production, J. Hydrol. 368(1-4): 79-87.

Kubiak J., 2003, Największe dimiktyczne jeziora Pomorza Zachodniego. Poziom trofii, podatność na degradację oraz warunki siedliskowe ichtiofauny (The largest dimictic lakes of Western Pomerania. Trophic status, susceptibility to degradation, and ichthyofauna habitat conditions), Rozpr. AR Szczec. 214, Szczecin, 96 pp (in Polish, English summary).

Kubiak J., Machula S., Stepanowska K., Biernaczyk. M., 2012, Evaluation of trophic level of the largest dimictic lakes of western Pomerania based on the Carlson criteria, Limnol. Rev. 12(2): 79-85.

Kubiak J., Tórz A., 2005, Eutrofizacja. Podstawowy problem ochrony jezior na Pomorzu Zachodnim (Eutrophication. Basic problems of lake conservation in Western Pomerania), Słup. Pr. Biol. 2: 17-36 (in Polish).

Kubiak J., Tórz A., 2006, Thermal regime of the biggest dimictic lakes of Western Pomerania region, Limnol. Rev. 6: 155-162.

Kudelska D., Cydzik D., Soszka H., 1994, Wytyczne monitoringu podstawowego jezior (Guidelines for the basic monitoring of lakes), Wydaw. PIOŚ, Warszawa, 42 pp (in Polish).

Lossow K., 1996, Rekultywacja jezior i zbiorników wodnych - dotychczasowe osiągniecia, możliwości i perspektywy (Recultivation of lakes and reservoirs - past achievements, opportunities and prospects), [in:] Ochrona i rekultywacja jezior i zbiorników wodnych (Protection and recultivation of lakes and reservoirs), Mat. II Konf. Nauk.-Tech., 7-8 March 1996, Międzyzdroje (Poland), Wydaw. Biuro Inf. Nauk., Szczecin: 47-56 (in Polish).

Lossow K., 1998, Ochrona i rekultywacja jezior - teoria i praktyka (Protection and recultivation of lakes - theory versus practice), Idee Ekolog. Ser. Szkice 13(7): 55-71 (in Polish, English summary).

[MGGP, IOŚ] Małopolska Grupa Geodezyjno Projektowa, Instytut Ochrony Środowiska, 2010, Projekt:
„Sformułowanie w warunkach korzystania z wód regionu wodnego ograniczeń w korzystaniu $\mathrm{z}$ wód jezior lub zbiorników oraz w użytkowaniu ich zlewni" (Project: "Formulation under conditions of use of waters of the water region of the restrictions on the water use from lakes or reservoirs and operation of their catchments") [typescript], NFOŚGW, Kraków-Warszawa, 1499 pp (in Polish).

Neal C., Heathwaite A.L., 2005, Nutrient mobility within river basins: a European perspective, J. Hydrol. 304(1-4): 477-490.

Olszewski P., 1971, Trofia a saprobia (Trophy and saproby), Zesz. Nauk WSR Olszt. Ser. C (Supl. 3): 5-14 (in Polish).

Olszewski P., Tadajewski A., 1959, Wpływ zlewni na żyzność jezior (Influence of catchment on lakes fertility), Zesz. Nauk. WSR Olszt. 4: 191-194 (in Polish).

Ptak M., Choiński A., Strzelczak A., Targosz A., 2013, Disappearance of Lake Jelenino since the end of the XVIII century as an effect of anthropogenic transformations of the natural environment, Pol. J. Environ. Stud. 22(1): 191-196.

Raczyński M., Waloch P., 2012, NATURA 2000: Standardowy formularz danych. Obszar - PLH320050 Dolina Tywy (NATURA 2000: Standard data form. Site - PLH320050 Dolina Tywy), GDOŚ, Warszawa, 7 pp (in Polish).

Sieńska J., Dunalska J., Łopata M., Parszuto K., Tandyrak R., 2016, Trophic state and recreational value of Lake Mikołajskie, Limnol. Rev. 16(3): 147-153.

Uchmański J., Szeligiewicz W., 1988, Empirical models for predicting water quality as applied to data on lakes of Poland, Ekol. Pol. 36: 3-4.

Vollenweider R., 1968, Scientific fundamentals of the eutrophication of lakes and following waters, with particular reference o nitrogen and phosphorus as factors in eutrophication, Tech. Rep. DAS/CSI/68.27, OECD, Paris, 150 pp.

Vollenweider R., 1976. Advances in defining critical loading level for phosphorus loading in lake eytrophication. Mem. Ist. Ital. Idrobiol. Dott. Marco Marchi 33: 53-83.

[WIOŚ] Wojewódzki Inspektorat Ochrony Środowiska w Szczecinie (Voivodship Inspectorate for Environmental Protection in Szczecin), 2016, Stan środowiska w województwie zachodniopomorskim w latach 2013-2015 (State of the environment in the West Pomeranian Voivodship in the years 2013-2015) [online], WIOŚ Szczecin, Szczecin, 366 pp (in Polish). Retrieved from https://www. wios.szczecin.pl/bip/chapter_16003.asp [accessed 16 May 2017].

Wołos A., Mioduszewska H., 2003, Wpływ stosowania przez wędkarzy zanęt na efekty wędkowania i bilans biogenów ekosystemów wodnych (Impact of ground-baits on angling effects and nutrients balance in aquatic ecosystems), Kom. Ryb. 1: 23-27 (in Polish). 